\title{
OR11-003 - The NLRP3 inflammasome is regulated by CaSR
}

\author{
JJ Chae ${ }^{1 *}$, G-S Lee ${ }^{1}$, N Subramanian², DL Kastner ${ }^{1}$ \\ From 7th Congress of International Society of Systemic Auto-Inflammatory Diseases (ISSAID) \\ Lausanne, Switerland. 22-26 May 2013
}

\section{Introduction}

Mutations in the gene encoding NLRP3 cause a spectrum of autoinflammatory diseases known as the cryopyrinassociated periodic syndromes (CAPS). NLRP3 is a key component of one of several distinct cytoplasmic multiprotein complexes (inflammasomes) that mediate the maturation of the proinflammatory cytokine interleukin$1 \beta$ (IL-1 $\beta$ ) by activating caspase- 1 . Although several models for inflammasome activation, such as $\mathrm{K}^{+}$efflux, generation of reactive oxygen species, and lysosomal destabilization have been proposed, the precise molecular mechanism of NLRP3 inflammasome activation, as well as the mechanism by which CAPS-associated mutations activate NLRP3, remains to be elucidated.

\section{Objectives}

To investigate how extracellular DAMP signals activate the NLRP3 inflammasome and the molecular pathogenesis of CAPS.

\section{Methods}

Using a combination of genetic, pharmacological, and biochemical approaches, we provide evidence that the CaSR is essential for NLRP3 inflammasome activation, which is directly controlled by intracellular $\mathrm{Ca}^{2+}$ and cAMP.

\section{Results}

$\mathrm{Ca}^{2+}$ or other CaSR agonists activate the NLRP3 inflammasome in the absence of exogenous ATP, whereas knockdown of CaSR reduces inflammasome activation in response to known NLRP3 activators. The CaSR activates the NLRP3 inflammasome through phospholipase $\mathrm{C}$ (PLC), which catalyzes inositol trisphosphate $\left(\mathrm{IP}_{3}\right)$ production and thereby induces release of $\mathrm{Ca}^{2+}$ from

${ }^{1}$ Medical Genetics Branch, National Human Genome Research Institute, Bethesda, USA

Full list of author information is available at the end of the article endoplasmic reticulum (ER) stores. The increased cytoplasmic $\mathrm{Ca}^{2+}$ promotes the assembly of inflammasome components, and intracellular $\mathrm{Ca}^{2+}$ is required for spontaneous inflammasome activity in cells from CAPS patients. CaSR stimulation also results in reduced intracellular cAMP, which independently activates the NLRP3 inflammasome. cAMP binds to NLRP3 directly to inhibit inflammasome assembly, and downregulation of cAMP relieves this inhibition. The binding affinity of cAMP for CAPS-associated mutant NLRP3 is substantially lower than for wild-type NLRP3, and the uncontrolled mature IL-1 $\beta$ production from CAPS patients' peripheral blood mononuclear cells is attenuated by increasing cAMP.

\section{Conclusion}

Taken together, these findings suggest that $\mathrm{Ca}^{2+}$ and cAMP are two key molecular regulators of the NLRP3 inflammasome that have critical roles in the molecular pathogenesis of CAPS. In addition, our data suggest a broader spectrum of potential targets for therapy of CAPS as well as other inflammatory conditions involving the NLRP3 inflammasome, including gout, type 2 diabetes mellitus, atherosclerosis, and Alzheimer's disease.

\section{Competing interests}

None declared.

\section{Authors' details}

${ }^{1}$ Medical Genetics Branch, National Human Genome Research Institute, Bethesda, USA. 'Laboratory of Systems Biology, National Institute of Allergy and Infectious Diseases, Bethesda, USA.

Published: 8 November 2013

doi:10.1186/1546-0096-11-S1-A192

Cite this article as: Chae et al:: OR11-003 - The NLRP3 inflammasome is regulated by CaSR. Pediatric Rheumatology 2013 11(Suppl 1):A192.
C Biomed Central

() 2013 Chae et al; licensee BioMed Central Ltd. This is an Open Access article distributed under the terms of the Creative Commons Attribution License (http://creativecommons.org/licenses/by/2.0), which permits unrestricted use, distribution, and reproduction in any medium, provided the original work is properly cited. 